DOI:10.2478/rrlm-2020-0029

\title{
Evaluation of oxidative stress in bladder in urethral injuries: an experimental model in rats
}

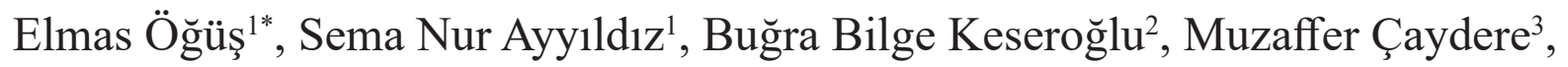 \\ Ali Ayyıldız², Doğan Yücel ${ }^{1}$
}
1. Department of Medical Biochemistry, Ankara Training and Research Hospital, University of Health Sciences, Ankara, Turkey
2. Department of Urology, Ankara Training and Research Hospital, University of Health Sciences, Ankara, Turkey
3. Department of Pathology, Ankara Training and Research Hospital, University of Health Sciences, Ankara, Turkey

\begin{abstract}
Background: The aim of this study is to determine the changes of some oxidative stress parameters following an experimental urethral injury. Materials and Methods: 24 Wistar-Albino male rats were used and 18 of them were exposed to urethral injury. Total oxidant status (TOS) and total antioxidant capacity (TAC) values in bladder and penile tissues and sera of rats during creation and healing stages of injury (control, day-1, 7 and 21) were examined and evaluated histopathologically. Results: TOS levels in both tissue and serum groups increased nearly $4 x$ fold in first day following injury. On the seventh day, TOS levels of bladder and penile tissue showed a nearly 50\% decrease according to first day ( $p=0.005 ; p=0.025$, respectively). At the end of third week, while bladder TOS levels decreased to control values, penile tissue TOS levels remained at high levels. The bladder TAC values mildly increased in first day $(p=0.026)$ and then returned to normal levels in seventh day and continued to decrease down by half of control levels at the end of third week $(p=0.005)$. TAC levels of penile tissue increased twofold in first day $(p=0.021)$ and remained at same high levels for three weeks. Conclusion: We observed that first day after traumatic urethral injury seems to be very important for evaluation of traumatic injuries and wound healing. We believe when an urethral injury was occured, both urethra and bladder should be handled with care and without delay during urethral healing, because of the presence of oxidative changes in bladder.
\end{abstract}

Keywords: urethral injury, bladder, total oxidant status, total antioxidant capacity, oxidative stress index Received: 30 th April 2020; Accepted: 30 ${ }^{\text {th }}$ June 2020; Published: $10^{\text {th }}$ July 2020

* Corresponding author: Elmas Öğüş, Department of Medical Biochemistry , Ankara Training and Research Hospital, University of Health Sciences, Ankara, 06230, Turkey. E-mail: oguselmas@gmail.com 


\section{Introduction}

Urethral injuries, which can be caused by biopsy, instrumentation, catheterization, diathermy, external trauma and urethritis, cause a scar tissue usually results in stricture and narrowing in the urethra (1). The degree of injury determines the severity of the narrowness to be formed. The results of urethral injury are mostly inflammation, ischemia, regeneration and fibrosis. Depending on the severity of the injury and reaction, healing and/or scarring of the tissue can be observed (2). Wound healing is a complex event. Factors such as extracellular matrix, cells, growth factors, and cytokines play a role in wound healing $(3,4)$. Oxidative stress has also been studied and shown to play a role in wound healing (5). Also, oxidative stress protects the body system by defending against pathological condition in the wound. While oxidative stress occurs in acute injuries, reactive oxygen species increase especially in chronic wounds (6). Oxidative stress is the deterioration of the balance between total oxidant status (TOS) and total antioxidant capacity (TAC), in favor of oxidant systems. Oxidative stress causes lipid peroxidation (7), protein oxidation (8) and DNA damage (9). Little is known about the presence of changes in the bladder in urethral injuries (2). If there are oxidative changes in bladder in urethral injuries, the focus should also be on the treatment of the bladder. Our aim is to determine the status of oxidative stress in the bladder and the level of exposure of the bladder in urethral injuries.

\section{Materials and Methods}

\section{Experimental study}

This study was conducted at the experimental animal laboratory after the approval of the ethics committee (26.05.2017/466) . A total of 24 Wistar-Albino male rats (200-255 g) were used. The rats were divided into four groups: control group ( $n=6)$, the first day group (day- $1, n=6)$, the first week group (day-7, $n=6$ ), and the third week group (day-21, $n=6$ ). The rats were housed in individual cages in a programmed temperature $\left(22-24^{\circ} \mathrm{C}\right)$, humidity $(50-55 \%)$ and light-dark cycle control controlled room $(12 \mathrm{~h} / 12 \mathrm{~h}$ of light and dark) with free access to food and water until the end of period for each group. Water was changed daily.

\section{Creation of urethral injury in rats and materi- al collection}

The rats were anaesthetized with a combination of ketamine-xylazine intraperitoneally before experiment. Urethral injury was created with the method previously described by Ayyıldız et al. (10). The control group was not intervened. In the other groups, an injury was created in the anterior-ventral urethra starting from approximately $0.5 \mathrm{~cm}$ proximal to the distal with a 29 $\mathrm{G}$ needle. The needle was hooked $90^{\circ}$ and the urethra was drawn four times from the same site to form urethrorrhagia. Urethral catheter was not inserted.

The rats were killed with an excessive dose of pentobarbital sodium $(100 \mathrm{mg} / \mathrm{kg}$, intraperitoneal) injection at the end of experiment. The control group was sacrified at the beginning of the study, while the other groups were sacrified at the end of the days $1 \mathrm{st}, 7 \mathrm{th}$ and $21 \mathrm{st}$. Blood samples $(\backsim 1.5 \mathrm{ml})$ were taken with cardiac puncture by using a $25 \mathrm{G}$ needle with $5 \mathrm{ml}$ syringe. Blood sera were separated following the resting of blood samples at room temperature for $30 \mathrm{~min}$ and centrifugation $(15 \mathrm{~min}$ at $3000 \times g)$ and stored at $-86^{\circ} \mathrm{C}$ until the time of analysis. The rats were dissected, the bladder tissues and the penile tissues containing urethra were removed. Halves of the tissue samples, after labeling, were immediately transferred to thermos bottle containing dry ice flakes and transported to biochemistry laboratory. The remaining parts were fixed in formalin solution and transported to pathology laboratory. 
Biochemical analysis of the tissue and serum samples were performed simultaneously. The samples were kept at $-86^{\circ} \mathrm{C}$ until the time of analysis.

\section{Biochemical evaluation}

$10 \mathrm{ml}$ of $140 \mathrm{mM} \mathrm{KCl}$ solution was added per gram of wet weight for the bladder or penile tissues, then all the tissues were homogenized. The homogenate was centrifuged at $3000 \mathrm{x}$ g at $4{ }^{\circ} \mathrm{C}$ for $10 \mathrm{~min}$. The resultant supernatant was used for the biochemical analyses.

Total oxidative status (TOS) as a marker of oxidative stress and total antioxidant capacity (TAC) as an indicator of antioxidant system were determined in the tissues and serum samples.

TOS and TAC measurements were performed in the Cobas c501 analyzer, with the automated methods using the Rel Assay Diagnostics kits (Turkey) described by Erel $(11,12)$.

In TOS measurement; the oxidants present in the sample oxidize the ferrous ion $\left(\mathrm{Fe}^{2+}\right)-$ chelator complex to ferric ion $\left(\mathrm{Fe}^{3+}\right)$. The ferric ion makes a colored complex with a chromogen in an acidic medium and is measured spectrophotometrically. Hydrogen peroxide was used as the calibrator and the total amounts of oxidant were expressed in $\mu$ mole hydrogen peroxide $\left(\mathrm{H}_{2} \mathrm{O}_{2}\right)$ equivalent/L.

In TAC measurement; the antioxidants in the sample reduce dark blue-green colored ABTS (2,2'-azino-bis-3-ethylbenzothiazoline-6-sulfonic acid) radical to its colorless form and the change of absorbance at $660 \mathrm{~nm}$ is related with total antioxidant level of the sample. Trolox, a water soluble form of vitamin E, was used as a calibrator and the results were expressed in mmole Trolox equivalent/L.

Because of the bladder and penile tissues were heterogenous and rich of fibro-muscular components, TOS and TAC levels of tissues were calculated per gram wet tissue instead of per mg protein, and TOS and TAC levels for serum samples were given per liter.
Oxidative stress index (OSI) was calculated as percent by using the formula;

$$
\begin{aligned}
\text { OSI }= & \left(\text { TOS, } \mu \text { mole } \mathrm{H}_{2} \mathrm{O}_{2} \text { equivalent } / \mathrm{L}\right) /(\text { TAC, } \\
& \text { mmole Trolox equivalent } / \mathrm{L}) \times 100 .
\end{aligned}
$$

\section{Histopathological evaluation}

Paraffin sections of the tissue samples were cut at a thickness of four micrometers and hematoxylin-eosin staining was applied after 24 hours of alcohol fixation. The tissue sections were examined with an Olympus BX-51 light microscope. All the samples were evaluated by the same pathologist (MÇ). The urethral samples were evaluated for inflammation, hyperemia-hemorrhage, epithelization, subepithelial and spongiosal body healing, total healing, and luminal narrowing. The bladder samples were evaluated for globe or collapsed state, inflammation, detrusor muscle changes, epithelial status, and hyperemia-hemorrhage. While scoring, "+" was considered as present and "-_" was considered as absent.

\section{Statistical analysis}

Statistical evaluation was performed with the SPSS $^{\circledR} 16$ statistical software.

TOS, TAC, and the calculated OSI data of groups were firstly analyzed with Kruskal-Wallis nonparametric test. Signifantly different paired groups were analyzed with nonparametric Mann-Whitney $U$ test. $p<0.05$ was considered significant.

\section{Results}

\section{Biochemical analysis}

TOS and TAC levels of all groups are given in Figure 1. The calculated OSI results of all groups are given in Table 1.

\section{a) TOS in tissues and serum}

There were significant differences among the TOS values between the paired groups in bladder (control vs. day-1 and control vs. day-7), in 


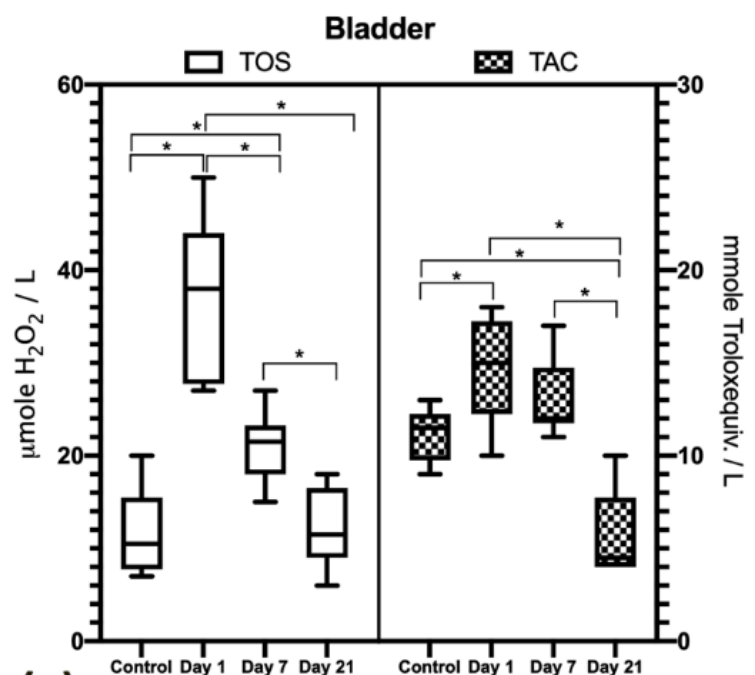

(a) Groups

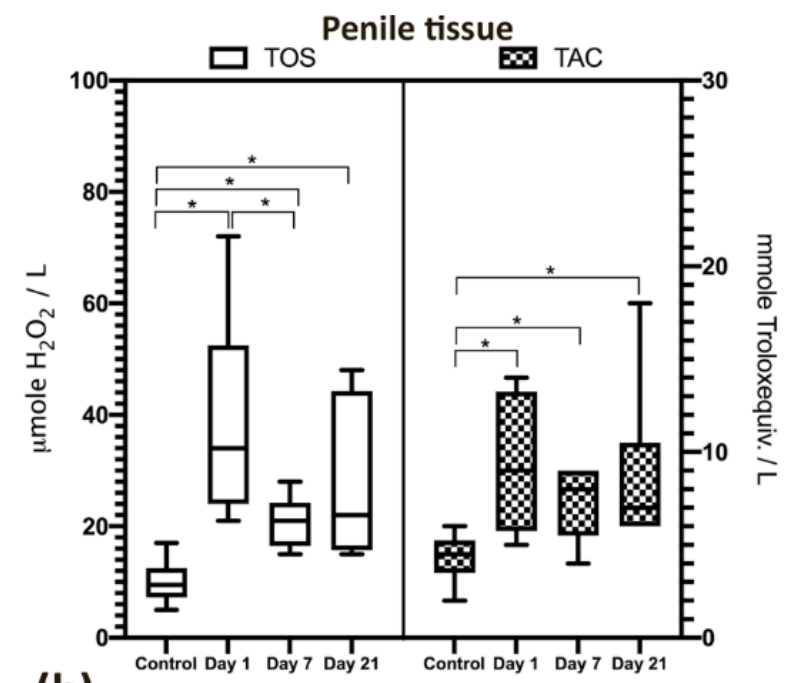

(b)

Groups

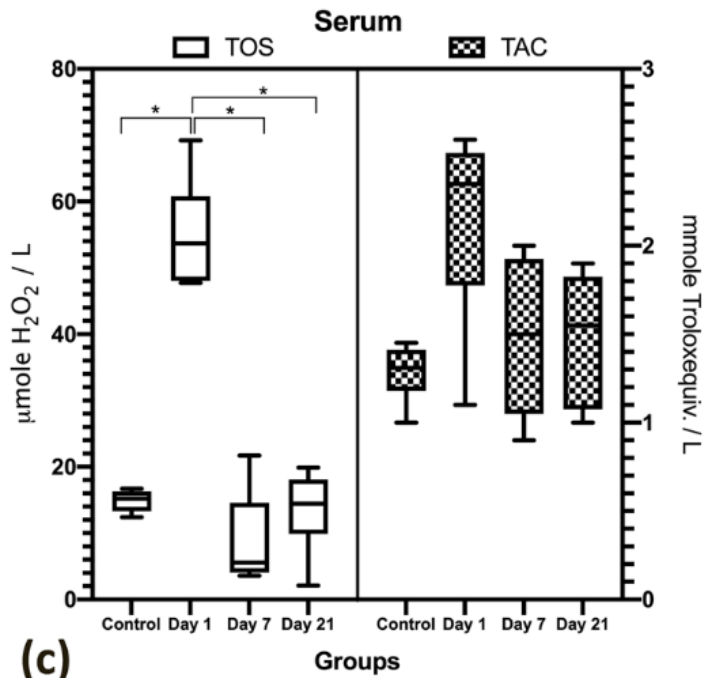

Fig, 1. Total oxidant status (TOS) and total antioxidant capacity (TAC) levels in (a) bladder and (b) penile tissues and (c) sera of the rats following urethral injury. Data are presented as box and whiskers plots thatshow the median and $25 / 75$ percentiles (box), and the minimum-maximum values (bars). Statistically significant differences between groups are labeled with an asterix, $(*)$.

penile tissues (control vs. day-1), and in serum (control vs. day-1, day-1 vs. day-7, and day-1 vs. day-21).

Experimental injury caused a nearly $4 \mathrm{x}$ fold increase of TOS levels in both tissue groups and serum in the first day. At the end of first week the TOS levels of bladder and penile tissue showed a nearly $50 \%$ decrease according to first day ( $p=0.005$ and $p=0.025$ respectively) but the reactions of bladder and penile tissues to injury were different in the third week. While the bladder TOS levels of day-21 decreased to control values $(p=0.810)$, the penile tissue TOS levels remained at high levels $(\mathrm{p}=0.013)$. 
Table 1. OSI in the bladder, penile tissues and serum

\begin{tabular}{lccccc}
\hline & \multicolumn{5}{c}{ Groups } \\
Median / & (Range) & p \\
\hline Parameter & $\begin{array}{c}\text { Control } \\
(n: 6)\end{array}$ & $\begin{array}{c}\text { Day-1 } \\
(n: 6)\end{array}$ & $\begin{array}{c}\text { Day-7 } \\
(n: 6)\end{array}$ & $\begin{array}{c}\text { Day-21 } \\
(n: 6)\end{array}$ & $\mathrm{M}^{-} \mathrm{W}^{*}$ \\
\hline Bladder & 0.99 & 2.81 & 1.59 & 1.96 & 0.002 \\
OSI & $(0.67-1.54)$ & $(1.56-3.13)$ & $(1.36-1.83)$ & $(1.50-4.50)$ & $\mathrm{a}, \mathrm{b}, \mathrm{c}, \mathrm{d}$ \\
\hline Penile tissue & 2.38 & 4.34 & 2.49 & 3.10 & 0.563 \\
OSI & $(1.33-3.40)$ & $(1.50-10.29)$ & $(1.88-7.01)$ & $(0.83-6.86)$ & - \\
\hline Serum & 1.21 & 2.59 & 0.33 & 1.05 & 0.004 \\
OSI & $(0.86-1.46)$ & $(1.85-4.33)$ & $(0.22-0.24)$ & $(0.11-1.75)$ & $\mathrm{a}, \mathrm{d}, \mathrm{e}$ \\
\hline
\end{tabular}

OSI; Oxidative stress index. $\mathrm{p}<0.05$ was considered statistically significant, M-W; Mann-Whitney $\mathrm{U}$ test.

${ }^{*}$ Letters below indicate statistically significant differences between paired groups:

$\mathrm{a}=$ Control vs Day-1, $\mathrm{b}=$ Control vs Day-7, $\mathrm{c}=$ Control vs Day-21, $\mathrm{d}=$ Day-1 vs Day-7,

$\mathrm{e}=$ Day-1 vs Day-21.

Serum TOS levels rapidly decreased after first day and remained at control levels in first and third weeks ( $p=0.04$ and $p=0.04$ respectively).

\section{b) TAC in tissues and serum}

The bladder TAC values mildly increased in the first day $(p=0,026)$ but returned to normal levels in day-7 and continued to decrease down to the half of the control at the end of the third week $(p=0.005)$. TAC levels of penile tissue increased twofold in the first day and remained at same high levels during the three weeks $(p=0.021)$.

The serum TAC values between groups were not significant in Kruskal-Wallis test $(\mathrm{p}=0.059)$.

\section{c) Oxidative Stress Index in the tissues and serum} The bladder OSI values showed a twofold increase in day-1 $(\mathrm{p}=0.004)$, and remained at high levels in the groups of day-7 and day-21 $(\mathrm{p}=0.041)$.

The penile OSI values among the penile tissue groups were not significant in Kruskal-Wallis test. There were significant differences in the OSI values of serum among the paired groups of serum (control vs. day-1, day-1 vs. day-7, and day1 vs. day-21 groups).

\section{Histopathology of the tissues}

In the histopathological evaluation, it was observed that the bladder collapsed on the first day after the urethral injury (Figure 2a). On the seventh day (day-7), the microscopical findings were replaced with a globe formation (Figure 2b). Also, an epithelial thinning, detrusor hypertrophy and marked inflammation were seen. Inflammation was shown in Figure 2c. At the end of experiment (day-21), there was no pathological findings except a mild inflammation. Epithelium and detrusor layer were normal (Table 2). In histopathological examination of urethra on the day-1 and day-7, evident inflammation, hyperemia and bleeding were found. The irregularity of the urethral epithelium, spongious tissue deterioration, and luminal narrowing also accompanied. In the last group of experiment (day-21), all the parameters except mild hyperemia were similar to control findings (Table 3 ).

\section{Discussion}

Oxidative stress is characterized by both acute and chronic episodes and it causes an increase in reactive oxygen species. Certain vital cell components, such as proteins, lipids and nucleic 


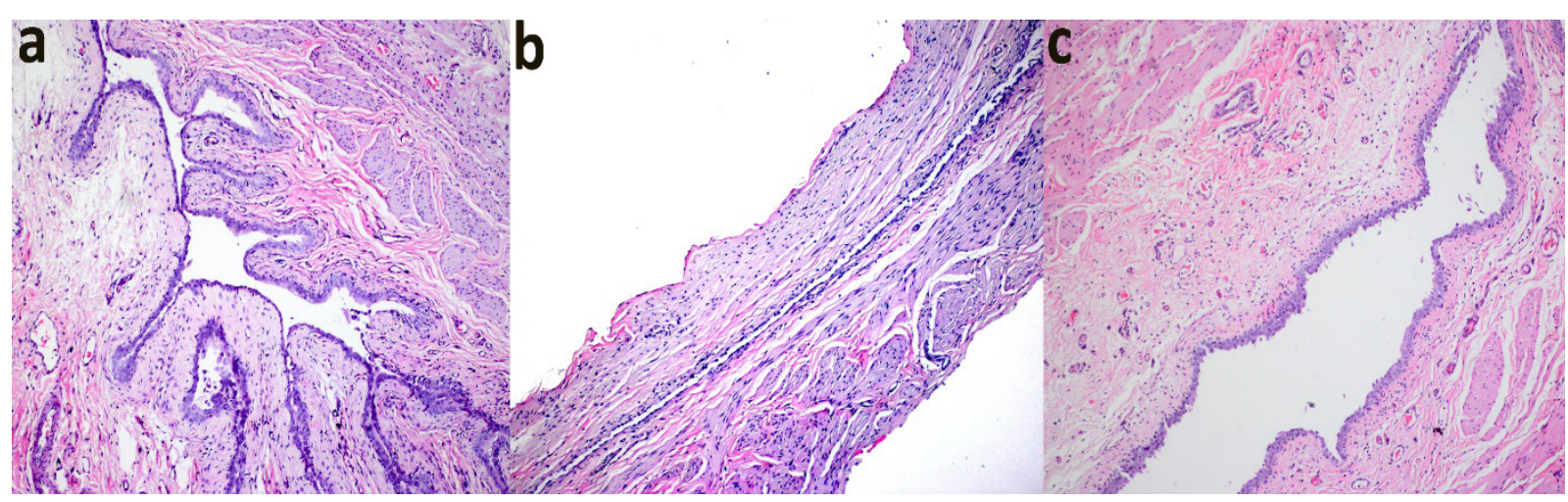

Fig. 2. Light microscopy of bladder tissues from rats (HE stained bladder section, 100x). (a) Collapse development (rat \#3 of first day group), (b) globe formation (rat \#5 of seventh day group), and (c) inflammation (rat \#6 of seventh day group).

Table 2. Histopatologic features in bladder tissue, $H \& E$

\begin{tabular}{lcccc}
\hline Presence of histopatologic features & Control $(\mathbf{n}=\mathbf{6})$ & Day $\mathbf{1}(\mathbf{n}=\mathbf{6})$ & Day 7 $(\mathbf{n}=\mathbf{6})$ & Day 21 $(\mathbf{n}=\mathbf{6})$ \\
\hline Collapse & 0 & 6 & 0 & 0 \\
\hline Globe & 0 & 0 & 5 & 0 \\
\hline Epithelial thinning & 0 & 0 & 6 & 0 \\
\hline Inflammation & 0 & 0 & 6 & 1 \\
\hline Detrussor hypertrophia & 0 & 0 & 5 & 0 \\
\hline
\end{tabular}

Table 3. Histopatologic features in urethra, $H \& E$

\begin{tabular}{llcccc}
\hline Histopatologic features & & Control $(\mathbf{n}=\mathbf{6})$ & Day $\mathbf{1}(\mathbf{n}=\mathbf{6})$ & Day $7(\mathbf{n}=\mathbf{6})$ & Day $21(\mathbf{n}=\mathbf{6})$ \\
\hline \multirow{2}{*}{ Coverage of urethral epithelium } & Complete & 6 & 5 & 4 & 6 \\
\cline { 2 - 6 } & Incomplete & 0 & 1 & 2 & 0 \\
\hline Urethral lumen narrowing & Presence & 0 & 2 & 1 & 0 \\
\hline Destruction spongious tissue & Presence & 0 & 2 & 2 & 0 \\
\hline Inflammation & Presence & 0 & 5 & 5 & 0 \\
\hline Total healing & Presence & 6 & 0 & 0 & 6 \\
\hline Hyperemia-Bleeding & Presence & 0 & 3 & 3 & 3 \\
\hline
\end{tabular}

acids, and consequently organs are affected and their functions are impaired (13).

In our study, TOS levels in bladder tissue were significantly higher than the control group on the first day according to TAC levels. So, OSI values were significantly higher in day-1, day-7 and day-21 compared to control group, indicating that there was an oxidant-antioxidant imbalance in these rats.

The statistical difference between day- 1 and day21 TAC levels in post-traumatic bladder tissue demonstrates that the recovery healed positively $(p=0.005)$. These findings are consistent with recovery findings and histopathological evaluation results. However, high levels of TAC are still present in the penile tissue containing the urethra on the 21 st day, indicating that the antioxidant effect continues against oxidative stress. More experimental and clinical studies with different experimental design are needed to elucidate the consequences of influencing the oxidant-antioxidant system in both bladder and 
serum when the bladder is not directly affected. Oxidative stress in the bladder leads to deterioration of mucous integrity. Free radicals destroy cell membranes, mitochondria, sarcoplasmic reticulum and synaptic membranes (14). However, it can be predicted that the occurrence of the bladder damage may be related to the duration of the damage to the urethra. Our study supports this statement. The TOS and TAC levels of the first and seventh days of injury return to normal levels at the end of third week. Histopathological evaluation also supports biochemical changes positively. However, on the first day of the injury, TOS values in bladder, penile tissue and serum increased at an advanced level, and decreased on the 7 th and 21 st days to control values. TAC levels in bladder, penile tissue and serum increased significantly but at a lower level. Although TAC levels in bladder and serum decreased to control values on the 21 st day, it remained significantly higher in penile tissue than the control group. According to our interpretation, despite the oxidation occuring in the bladder after injury, the antioxidant system existing in the rat's body decreases in the third week. It may be useful to pay attention to oxidative stress in the bladder, especially in those with frequent or previous urethral injuries. Antioxidant treatments can prevent inflammation in the bladder and associated oxidative reactions and damage in patients who experience urethral trauma many times.

In this study, serum oxidative stress index values were found to be higher in the day 1 group (OSI $=2.74)$ than in the control group (OSI $=1.18)$, and decreased in the first week group (OSI = $0.78)$. In the third week, values close to the first week values were observed (OSI $=1.03)$. This may be due to changes in leaking factors during tissue healing.

The collapse in the bladder on the first day may be due to the oxidant-antioxidant system change which develops in both the urethra and the blad- der due to urethral injury. It may also be a reflex event to prevent the urine from passing through the urethra. This reflex may have been triggered by an oxidant-antioxidant system. We do not know how long the collapse of the bladder lasted. After the urethral injury, it is possible to develop only dysuria and urethral pain, and acute urinary retention may also occur due to the degree and severity of injury. Acute urinary retention is associated with bladder ischemia (13). Often an urethral catheter is used to cure this condition. In our study, urine retention was not observed in the bladder one day after the urethral injury, but the bladder collapsed. The collapse of the bladder can be based on a number of reasons, including the reflex mechanism. However, TOS and TAC levels in the bladder tissue of the day-1 rised at a higher level. Kirpatovsky et al. showed that oxidative stress is supressed when antioxidants are used in cases of acute urinary retention (13). The relationship between increased oxidative stress and bladder collapse is unclear, and further work is required.

It has been confirmed that the functional impairment of the urinary bladder following chronic outlet obstruction and acute overdistention might come from tissue ischemia and ischemia/ reperfusion injury (15). Free radical scavengers, antioxidants, or materials inhibiting ischemia/ reperfusion injury may diminish bladder damages caused by bladder output obstruction or overdistention. Studies show that oxidative stress caused by bladder ischemia and ischemia/reperfusion injury affects bladder functions $(13,15)$. However, in our study, although there was a minimal anterior urethra injury and bladder ischemia and ischemia/reperfusion injury did not develop, the same mechanism was activated and the oxidative stress occured in the bladder, which we believe developed due to inflammation. It is observed that serum TOS values increased suddenly on the first day, then decreased below normal levels on the 7 th day and approached normal 
levels on the 21 st day. Injury to the urethra will probably lead to a change in the oxidant/antioxidant values of the whole system, including the kidneys, not just the bladder. Further studies are needed to support this theory. In our study, oxidative stress increased in the bladder after urethral injury. While the oxidative stress, which results from an injury, continued to increase until the third week in the penile tissue, it decreased to the control level in the third week in the bladder. In contrast, serum oxidative stress index levels were highest in the day 1 group and lowest in the first and third week.

In this study, we think that oxidative stress in the bladder is triggered by inflammation. Urethral pain and dysuria occur in the first urine outlet after urethral injury. As a result, the collapse might be occured on the first day reflexively to decrease the subsequent amounts of urine. Globe formation on the 7th day may be a result of the oxidant/antioxidant system, which changes from the first day to the 7 th day. The oxidative stress mechanism that develops in the bladder after urethral injury depends on the deterioration of the balance between the endogenous and exogenous antioxidants, the pro-oxidative system, free radicals, and the reactive oxygen species and reactive nitrogen species systems. With prolonged healing in the urethra, an increase in oxidative stress and inflammation will occur as the situation will change in the favor of these systems. This may lead to an abnormal increase in factors associated with transcription, growth and apoptosis (16).

If this vicious cycle continues, that is, if the healing of the urethra continues for a long time, the mechanism predicted above will proceed to an advanced stage causing apoptosis, fibrosis and denervation in the bladder, and structural and functional changes will occur (16). In this study, urethral damage is minimal and according to our information, healing of the urethra begins 72 hours after injury (4). Therefore, it is necessary to evaluate the results of this study as minimal urethral injury.

There are also limitations in our study. First, only the urethra could be studied instead of the entire penile tissue. However, it was not technically easy to isolate the urethra of the rat and to study only oxidative stress markers in the urethral tissue. Second, the oxidant/antioxidant status in the urethra could be demonstrated using immunohistochemical staining showing oxidative stress markers. We were able to make a limited evaluation due to the analysis of tissue sections only with basic H\&E staining. These limitations can be explored further in future studies.

\section{Conclusion}

In this study, we have observed that the first day following traumatic urethral injury seems to be very important for the evaluation of traumatic injuries and wound healing. In the late phase of trauma and healing process (two or more weeks after trauma), findings about oxidative stress in bladder may not be dramatic as in the first day. In conclusion, we believe that when an urethral injury is occured, both of the urethra and the bladder should be treated with care during urethral healing, because of the presence of oxidative changes in the bladder.

\section{Abbreviations \\ TAC: Total Antioxidant Capacity \\ TOS: Total Oxidant Status \\ OSI: Oxidative Stress Index}

\section{Authors' contribution}

EÖ-AA: Conceptualization; investigation, original draft preparation; Writing- original draft; Writing -review \& editing EÖ-SNA-BBK-MÇ: methodology, formal analysis, investigation 


\section{EÖ-AA-DY: review and editing, visualiza- tion, project administration, and funding acquisition}

\section{Conflict of interest}

There are no conflicts of interest.

\section{References}

1. Verla W, Oosterlinck W, Spinoit AF, Waterloos M. A comprehensive review emphasizing anatomy, etiology, diagnosis, and treatment of male urethral stricture disease. Biomed Res Int. 2019. doi: 10.1155/2019/9046430.

2. Wynn TA. Cellular and molecular mechanisms of fibrosis. J Pathol. 2008; 214(2): 199-210. doi: 10.1002/ path. 2277.

3. Da-Silva EA, Sampaio FJ, Dornas MC, Damiao R, Cardoso LE. Extracellular matrix changes in urethral stricture disease. J Urol. 2002;168(2):805-807.

4. Kumar S, Wong PF, Leaper DJ. What is new in wound healing? Turk J Med Sci. 2004;34:147-160.

5. Sen CK, Khanna S, Gordillo G, Bagchi D, Bagchi M, Roy S. Oxygen, Oxidants, and Antioxidants in Wound Healing-An Emerging Paradigm. Ann. N.Y. Acad. Sci. 2002;957:239-249.

6. Gordillo GM, Sen CK. Revisiting the essential role of oxygen in wound healing. Am J Surg. 2003;186(3):259263.

7. Halliwell B, Gutteridge JM. Lipid peroxidation, $\mathrm{O} 2$ radicals, cell damage and antioxidant therapy. Lancet 1984;1(8391):1396-1397.

8. Tamarit J, Cabiscol E, Ros J. Identification of the ma- jor oxidatively damaged proteins in E. coli exposed to oxidative stress. J Biol Chem. 1998;273(5):3027-3032.

9. Dizdaroglu M, Jaruga P. Mechanisms of free radical-induced damage to DNA. Free Radic Res. 2012;46(4):382419.

10. Ayyıldız A, Akgül KT, Cebeci Ö, Nuhoğlu B, Çaydere M, Üstün $H$, et al. Intraurethral honey application for urethral injury: An experimental study. Int Urol Nephrol. 2007; 39(4): 815-821.

11. Erel O. A novel automated method to measure total antioxidant response againts potent free radical reactions. Clin Biochem. 2004;37(4):112-119.

12.Erel O. A new automated colorimetric method for measuring total oxidant status. Clin Biochem. 2005;38(12):1103-1111.

13. Kirpatovsky VI, Plotnikov EY, Mudraya IS, Golovanov SA, Drozhzheva VV, Khromov RA, et al. Role of oxidative stress and mitochondria in onset of urinary bladder dysfunction under acute urine retention. Biochemistry (Mosc). 2013;78(5):542-548.

14. Malone L, Schuler C, Leggett RE, Levin RM. The effect of in vitro oxidative stress on the female rabbit bladder contractile response and antioxidant levels. ISRN Urol. 2013:639685. doi: 10.1155/2013/639685.

15. Lin AT, Juan YS. Ischemia, Hypoxia and Oxidative Stress in Bladder Outlet Obstruction and Bladder Overdistention Injury. Low Urin Tract Symptoms. 2012;4:Suppl 1, 27-31. doi: 10.1111/j.1757-5672.2011.00134.x.

16. Miyata Y, Matsuo T, Mitsunari K, Asai A, Ohba K, Sakai H. A review of oxidative stress and urinary dysfunction caused by bladder outlet obstruction and treatments using antioxidants. Antioxidants 2019, 8, 132. doi: 10.3390/antiox 8050132 . 